\title{
A reputação do provedor de serviços e os custos de troca como determinantes da retenção de clientes de um plano de saúde
}

\author{
The reputation of the services provider and the switching costs as determinants \\ of customer retention a health plan
}

\author{
Luciene Eberle ${ }^{1}$ \\ Gabriel Sperandio Milan ${ }^{2}$ \\ Deonir De Toni ${ }^{3}$
}

\begin{abstract}
Resumo
A retenção de clientes tem sido um tema significativo na literatura que trata das trocas relacionais em provedores de serviços. O presente estudo objetivou desenvolver e testar um modelo teórico contemplando os construtos reputação do provedor de serviços e a custos de troca como determinantes da retenção de clientes. Para tanto, foi aplicada um pesquisa quantitativa descritiva, por meio de uma survey a uma amostra de 269 empresas-clientes de uma operadora de planos de saúde. Os resultados foram analisados por meio da modelagem de equações estruturais e a mediação foi testada pelo procedimento de bootstraping. Os resultados comprovam que a retenção de clientes é influenciada diretamente e positivamente pela reputação do provedor de serviços e os custos de troca. Porém, não se confirmou a mediação dos custos de troca entre a reputação do provedor de serviços e a retenção de clientes.
\end{abstract}

Palavras-chave: Reputação do Provedor de Serviços; Custos de Troca; Retenção de Clientes; Trocas Relacionais.

\begin{abstract}
Customer retention has been a significant theme in the literature on the relational exchange in service providers. This study aimed to develop and test a theoretical model considering the constructs: the reputation of the services provider and switching costs as determinants of customer retention. To that end, a descriptive and quantitative research was applied through a survey to 269 client companies of a health plans operator. The results were analyzed using structural equation modeling and mediation was tested using a bootstrapping procedure. The results show that customer retention is directly and positively influenced by the reputation of the service provider and switching costs. Though, it was not confirmed the mediation of switching costs between the reputation of the service provider and customer retention.
\end{abstract}

Keywords: Reputation of the Service Provider; Switching Costs; Customer Retention; Relational Exchanges.

\section{Introdução}

De acordo com Aurier e N'Goala (2010), o objetivo central da retenção de clientes é aprofundar e ampliar as relações existentes, de modo que os clientes se sintam progressivamente desencorajados a trocar de parceiros de negócio. Para tanto, Palmatier et al. (2006) destacam que o desenvolvimento de vínculos estruturais permite que as empresas de serviços alcancem uma diferenciação nos mercados onde

Doutora em Administração pela Associação Ampla Pontifícia Universidade Católica do Rio Grande do Sul, PUCRS e Universidade de Caxias do Sul, UCS (2014). Mestre em Administração pela UCS - Universidade de Caxias do Sul (2009). Afiliação: Universidade de Caxias do Sul - UCS. Brasil. Lattes: http://lattes.cnpq.br/9510755031322359 Orcid: http://orcid.org/0000-0002-0282-5626 Email: leberle@ucs.br

2 Pós-Doutor em Administração na área de Marketing pela UFRGS - Universidade Federal do Rio Grande do Sul (2016). Doutor em Engenharia de Produção na área de Sistemas de Qualidade pela UFRGS (2006). Mestre em Engenharia de Produção na área de Qualidade e Gerência de Serviços pela UFRGS (2000). Afiliação: Universidade de Caxias do Sul - UCS Brasil. Lattes: http://lattes.cnpq.br/1063672354605352 Orcid: http://orcid.org/0000-0003-3480-2653 Email: gasmilan@ucs.br

3 Pós-Doutorado em Administração pela Universidade de São Paulo, Doutorado em Administração pela Universidade Federal do Rio Grande do Sul, Mestrado em Administração pela Universidade Federal do Rio Grande do Sul. Afiliação: Universidade de Caxias do Sul - UCS. Brasil. Lattes: http://lattes.cnpq.br/9042437952029909 Orcid: http://orcid.org/0000-0001-9637-8099 Email: deonirdt@terra.com.br 
estão inseridas, permitindo que obtenham possíveis vantagens competitivas sustentáveis e melhorem seu desempenho econômico-financeiro. Porém, os autores concluíram que a literatura não fornece definições completas e claras dos processos psicológicos que fundamentam as decisões dos clientes em manter, desenvolver e ampliar os relacionamentos com os provedores de serviços, especificamente.

Outra suposição é que a retenção de clientes não está relacionada apenas a comportamentos definitivos, e sim a indicativos da direção provável de comportamentos futuros (ROOS; GUSTAFSSON, 2007), relacionados à repetição de negócios (REICHHELD, 1996). É importante salientar que, ao proceder a um levantamento das recomendações de pesquisas futuras, nas principais bases de pesquisa que abordam os construtos investigados, ainda são percebidas lacunas ao se tratar da retenção de clientes e seus determinantes em empresas prestadoras de serviços, como forma de possibilitar o aumento da lucratividade e da rentabilidade do negócio (N'GOALA, 2007; JONES et al., 2007; BRUSH; DANGOL; O'BRIEN, 2012) e da competitividade das empresas (MILAN; DE TONI, 2012).

Em se tratando dos serviços de saúde, os estudos sobre a retenção de clientes destacam que as empresas prestadoras de serviços devem criar barreiras à perda (ou deserção) de clientes, principalmente por meio do desenvolvimento de relacionamentos interpessoais fortes, destacando a falta de eficiência dos fornecedores alternativos e os custos de troca ou sanções financeiras às deserções. Em outras palavras, mesmo que um cliente não esteja totalmente satisfeito com um determinado provedor de serviços, acabará mantendo o relacionamento com a empresa, pois os custos de troca são percebidos, geralmente, como elevados (PATTERSON, 2004).

De acordo com Jones et al. (2007), pesquisas futuras devem concentrar os esforços em testar os custos de troca, bem como outros determinantes, por exemplo, a reputação do provedor de serviços, e seu impacto sobre a retenção de clientes. Com a intenção de ampliar a discussão sobre a temática dos custos de troca, percebe-se que, quanto maior a intangibilidade e a heterogeneidade dos serviços, maior é a possibilidade de incidência da reputação e dos custos de troca na retenção de clientes (BURNHAM; FRELS; MAHAJAN, 2003; HODOVIC-BABIC; MEHIC; ARLANAGIC, 2011).

Levando em consideração as indicações de desenvolvimento de estudos futuros identificadas, a presente pesquisa foi desenvolvida com o objetivo de melhor compreender a relação existente entre um provedor de serviços e seus clientes corporativos, ou seja, no âmbito B2B - Business-to-Business, a partir da verificação da relação entre os construtos Reputação do Provedor de Serviços e os Custos de Troca como determinantes da Retenção de Clientes.

\section{Referencial Teórico e Relações Hipotetizadas}

De acordo com o objetivo proposto para a pesquisa, são apresentados os construtos determinantes (ou antecedentes) da retenção de clientes e as respectivas relações hipotetizadas. Para tanto, é apresentada a conceituação da reputação do provedor de serviços e dos custos de troca como determinantes da retenção de clientes e suas possíveis relações.

\subsection{Reputação do provedor de serviços}

O primeiro determinante analisado foi a reputação do provedor de serviços, o qual Barnett, Jermier e Laffert (2006) destacam que há maneiras diferentes de se entender tal construto, pois a reputação pode ser representada como sendo um sinal, uma imagem, uma identidade ou um ativo intangível (HODOVIC-BABIC; MEHIC; ARSLANAGIC, 2011). De outra forma, a reputação, muitas vezes, é representada como estando associada a uma identidade corporativa (conjunto de símbolos), a uma imagem corporativa (impressões sobre a empresa), ou seja, uma reputação corporativa (na perspectiva dos stakeholders) e um capital intangível relativo aos seus recursos e capacidade de gerar riquezas (PHILIPPE; DURAND, 2011).

Por sua vez, Hodovic-Babic, Mehic e Arlanagic (2011) afirmam que uma boa reputação traz uma série de benefícios estratégicos para as empresas, como, por exemplo, a redução de custos, a possibilidade de praticar preços especiais (preços premium), o interesse de novos investidores e/ou clientes, além de aumentar a rentabilidade através da criação de barreiras à troca do cliente para a concorrência (FOMBRUN, 
1996; ANG; WIGHT, 2009). E isto ajuda os clientes a tomar melhores decisões em situações nas quais não é possível avaliar a qualidade antes da compra, como é o caso dos serviços (HELM, 2007).

O estudo de Graham e Bansal (2007) também procurou examinar os determinantes das percepções dos clientes em relação à reputação do provedor de serviços, mais especificamente de uma companhia aérea, procurando entender como a reputação pode influenciar nas decisões dos clientes, as quais representaram as suas vontades. Neste contexto, os autores obtiveram duas contribuições fundamentais: uma é bem sugestiva, pois destaca que os clientes geralmente avaliam boas reputações quando são atendidas as promessas realizadas pelo provedor de serviços, enquanto que a outra sinaliza que a reputação afeta, de forma elevada, o desempenho organizacional (vide, também, WALKER, 2010; TYRAN; GIBSON, 2008).

Além disso, a reputação corporativa é uma construção atitudinal, desenvolvida sobre as interações e experiências, associada à sustentabilidade, o desempenho ampliado e o maior rendimento para as empresas e para os clientes. Pode-se afirmar ainda que, a reputação corporativa refere-se a um recurso estratégico, o qual contribui para uma vantagem competitiva contínua (GÖZÜKARA; YILDIRIM, 2015).

Outro aspecto fundamental da reputação, é que a mesma pode ser entendida como um julgamento coletivo, relativamente estável e de longo prazo, construída por várias partes interessadas $(\mathrm{OU}$; $\mathrm{SHIH}$; CHEN, 2014). Nesse sentido, Clemes, Gan e Zhang (2010) destacam que uma boa reputação desempenha um papel importante na continuidade dos relacionamentos com os parceiros de negócios e com os clientes.

\subsection{Custos de troca}

Por outro lado, os custos de troca despontam como determinante da retenção de clientes. Devido à concorrência acirrada no mundo dos negócios, as empresas devem se esforçar para evitar a perda ou a deserção de seus clientes (REICHHELD, 1996). Portanto, um dos desafios críticos é o de entender porque os clientes mudam de fornecedor ou de provedor de serviços. Sendo assim, verifica-se que os pesquisadores têm estudado e sugerido maior ênfase no entendimento e nos comportamentos e nas intenções associados aos custos de troca (VASUDEVAN; GAUR; SHINDE, 2006; KIM; AHN, 2006).

Seguindo nesta linha de raciocínio, os custos de troca podem ser entendidos como a percepção do cliente em relação à magnitude das despesas adicionais que direcionam para o término da relação atual e visam afiançar um fornecedor ou provedor de serviços alternativo. Estas despesas podem ser medidas em condições monetárias e em condições psicológicas de se tornar cliente de uma nova empresa, considerando o tempo e o esforço envolvidos em um novo relacionamento (BURNHAM; FRELS; MAHAJAN, 2003). Os custos de troca se referem a todos os custos que o cliente deve suportar quando decide mudar de um fornecedor ou provedor de serviços atual para outro alternativo. Porém, alguns autores destacam que há lacunas entre o entendimento teórico e o entendimento empírico ao se tratar de pesquisas acerca dos custos de troca (GRZYBOWSKI, 2008; GÓMEZ; MAÍCAS, 2011).

Os custos de troca envolvidos em ambientes de serviços são dinâmicos devido à interação entre a empresa e o cliente, considerando que, normalmente, os provedores de serviços são mais próximos de seus clientes e que, por isso, possuem mais oportunidades de oferecer algo especial que impacte na mente dos clientes (SIERRA; McQUITTY, 2005), até porque as relações de longo prazo são a chave da sobrevivência e de uma possível fonte de vantagem competitiva em mercados de alta concorrência

Contudo, baseados na observação do comportamento do parceiro de negócios, Johnson e Grayson (2005) sinalizam uma ligação entre a reputação e os custos de troca. Em se tratando de um provedor de serviços, uma reputação elevada tende a aumentar os custos de troca e, consequentemente, a evitar a deserção de clientes (WALSH; DINNIE; WIEDMAN, 2006). Porém, Walsh, Dinnie e Wiedman (2006) reforçam a necessidade de se desenvolver novos estudos que testem a relação direta e positiva da reputação sobre os custos de troca, por não haver, ainda, evidências empíricas definitivas.

Considerando a dificuldade de se definir a reputação empresarial, ou corporativa, apontada na literatura, Santos e Fernandes (2008) também trazem como indicação de desenvolvimento de pesquisas futuras que se verifique sua relação com os custos de troca, em diferentes contextos organizacionais, visando identificar 
a força da reputação do provedor de serviços em relação aos seus clientes e ao mercado no qual a empresa está inserida. Diante do exposto, foi formulada a primeira hipótese de pesquisa:

$H_{1}$ : A reputação do provedor de serviços influencia positivamente os custos de troca.

\subsection{Retenção de clientes}

Ampliando a discussão, verifica-se que a reputação é construída com base nas experiências diretas dos clientes ou consumidores com certa empresa, bem como por meio de formas indiretas de comunicação que fornecem informações sobre esta empresa (AULA; MANTERE, 2013). Da mesma forma, verifica-se que as operadoras de planos de saúde concorrem, muitas vezes, não apenas pelos serviços ofertados ou pelos seus preços, mas pela sua reputação (RIVERS; GLOVER, 2008). Assim, empresas de saúde, com imagem positiva sobre seus serviços, podem alcançar uma diferenciação, balizada no alto conhecimento, na repetição das escolhas de seus clientes (retenção de clientes) e em uma reputação destacada perante a concorrência (CHACHAL; BALA, 2012).

Além disso, Helgesen e Nesset (2007) testaram a relação da reputação como direcionadora para a retenção de clientes, e obtiveram como resultado que a reputação do provedor de serviços reflete o grau pelo qual as experiências de compra ou de consumo (utilização) aumentam a consistência das experiências dos clientes e, com o passar do tempo, ajudam a fomentar a retenção dos clientes. Neste contexto, o estudo de Walsh, Dinnie e Wiedmann (2006) aponta como implicações futuras de pesquisa, que se deve procurar por um melhor entendimento entre esta relação e, para isso, sugerem a aplicação de pesquisas empíricas em diversos contextos de serviços.

Considerando isso, as empresas prestadoras de serviços deveriam considerar a construção, a manutenção e a consolidação de sua própria reputação como um aspecto estratégico fundamental (FOMBRUN, 1996), pois influencia diretamente a percepção dos clientes quanto à sua permanência em um relacionamento sob uma perspectiva de longo prazo (LOUREIRO; KASDTENHOLZ, 2011; MILAN; DE TONI, 2012; MILAN et al., 2013). Sendo assim, Gözükara e Yildirim (2015) reforçam que uma reputação corporativa favorável leva as organizações a ampliar a retenção de clientes e, consequentemente, o crescimento econômico. Dessa forma, apresenta-se a segunda hipótese de pesquisa:

$H_{2}$ : A reputação do provedor de serviços influencia positivamente a retenção de clientes.

Ampliando a discussão, a pesquisa de Jones et al. (2007) destaca a importância dos custos de troca ao se definir as estratégias relativas à retenção de clientes, visando o encorajamento do cliente em manter os relacionamentos com os provedores de serviços atuais (BURNHAM; FRELS; MAHAJAN, 2003; ROOS; GUSTAFSSON, 2007), enfrentando, assim, mais desafios ou barreiras para trocar de provedor de serviços, até porque os clientes não mudam de empresa tão facilmente. Complementando, os custos de troca representam um desincentivo para explorar novos fornecedores de serviços e tem um impacto positivo na continuidade dos relacionamentos com os clientes de longo prazo (OU; SHIH; CHEN, 2014).

Desse modo, a questão que orienta o estudo de N'Goala (2007) é como a retenção de clientes pode ser mantida, considerando o fato de como os clientes podem ser impedidos de trocar de provedor de serviços quando não se sentem totalmente envolvidos no relacionamento cliente-empresa. Uma vez que um provedor de serviços foi escolhido, os clientes não gostariam de substituí-lo, a menos que ocorra uma experiência negativa, insatisfatória, ou uma ação específica da concorrência, pois os custos de troca se configuram como barreiras à interrupção do relacionamento constituído (WONG, 2011; KONUK; KONUK, 2013).

De acordo com Brush, Dangol e O'Brien (2012), os clientes são mais sensíveis aos custos de troca quando investem considerável tempo e esforço para desenvolver capacidades e relacionamentos necessários para otimizar a utilização de um determinado produto e/ou serviço, sendo que estas capacidades não podem ser transferidas e, a partir disso, as empresas obtêm vantagens comparativas ou mesmo competitivas por meio da exploração dos custos de troca. Diante disso, quando os clientes contratam e utilizam serviços de uma mesma empresa, a fim de evitar os custos associados à troca, os lucros da empresa aumentarão. 
Em acréscimo, os resultados do estudo desenvolvido por Seo, Ranganathan e Babad (2008) demonstram que há uma forte relação entre os custos de troca e a retenção de clientes. Com a intenção de ampliar o entendimento desta relação, os autores sugerem que sejam aplicados novos estudos considerando outros contextos de serviços, para que se consiga entender de forma mais ampla o comportamento entre estes dois construtos. Consoante isso, emerge a terceira hipótese de pesquisa:

$H_{3}$ : Os custos de troca influenciam positivamente a retenção de clientes.

Para facilitar a compreensão acerca do modelo teórico testado, bem com das respectivas hipóteses de pesquisa, segue a Figura 1.

Figura 1 - Modelo teórico proposto

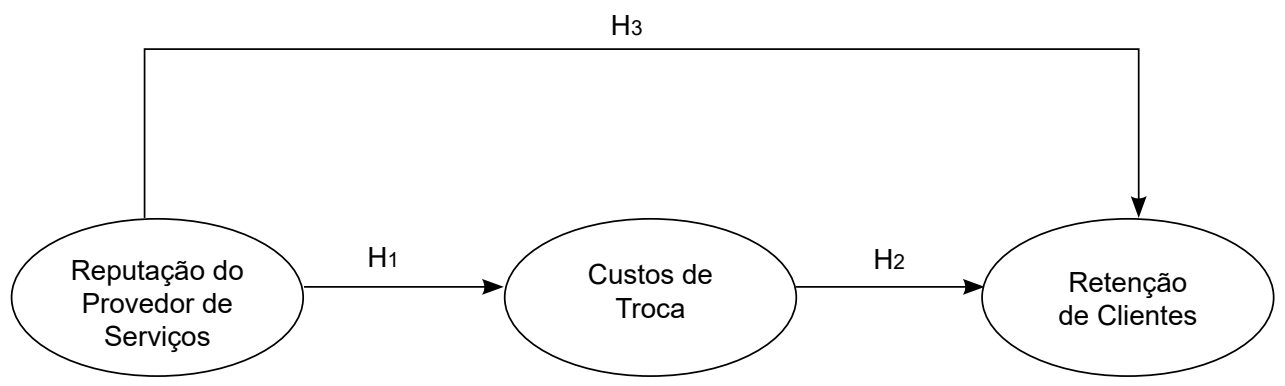

Fonte: Elaborada pelos autores.

Com base no pressuposto defendido por Palmatier et al. (2006), de que os custos de troca podem ajudar a explicar os efeitos mediadores relacionais, sendo compreendidos como fatores chave na perspectiva dos clientes, optou-se, também, por testar o efeito indireto, portanto, de mediação (HAYES, 2013; JOSE, 2013), dos custos de troca entre a reputação do provedor de serviços e a retenção de clientes. Desse modo, surgiu a quarta hipótese de pesquisa:

$H_{4}$ : Os custos de troca são mediadores da relação entre a reputação do provedor de serviços e a retenção de clientes.

\section{Método de pesquisa}

Na presente pesquisa, foi utilizado o método quantitativo descritivo, por meio da aplicação de uma survey (levantamento) de corte transversal, implementada com base em um questionário estruturado (HAIR Jr. et al., 2009; FINK, 2013). Para a análise dos dados foi utilizada diversas técnicas e procedimentos multivariados, mais especificamente, a aplicação da técnica de Modelagem de Equações Estruturais (BYRNE, 2010; KLINE, 2011; HOYLE, 2012; BLUNCH, 2013).

Como ambiência de pesquisa foi utilizada os planos coletivos (empresariais) de uma operadora de serviços da saúde, correspondendo a uma população de 3.128 clientes (empresas). Para tanto, destacase que ao se definir os participantes da pesquisa, levou-se em conta a técnica dos informantes-chave (JAWORSKI; KOHLI, 1993). Neste caso, sendo eles caracterizados como executivos ou gestores das áreas de recursos humanos ou administrativo-financeira, os quais são os responsáveis pelas negociações dos contratos de planos de saúde em suas empresas.

Considerando os critérios definidos para a pesquisa, foi determinada uma amostra aleatória, realizada por meio de sorteio, a qual totalizou 286 casos, tendo em vista a utilização do método de equações estruturais e de sua complexidade, os quais sugerem que a amostra seja composta por mais de 200 casos válidos (KLINE, 2011; BYRNE, 2010).

$\mathrm{Na}$ elaboração do questionário de pesquisa, foi adotada uma escala intervalar do tipo Likert de sete pontos para todas as questões, tendo em seus extremos "1. Discordo Totalmente" a "7. Concordo Totalmente". Destaca-se que as escalas utilizadas foram operacionalizadas a partir de estudos anteriores, conforme mostra a Tabela 1. 
Tabela 1 - Operacionalização dos construtos.

\begin{tabular}{ll|l}
\hline Construtos & Variáveis & Autores \\
\hline $\begin{array}{l}\text { Reputação do Provedor } \\
\text { de Serviços }\end{array}$ & $\begin{array}{l}\text { REPUT_1 a 3 e 5 } \\
\text { REPUT_4 }\end{array}$ & $\begin{array}{l}\text { Ganesan (1994) e Doney e Cannon (1997) } \\
\text { Milan e De Toni (2012) e Milan et al. (2013) }\end{array}$ \\
\hline Custos de Troca & CUSTR_1 a 3 & $\begin{array}{l}\text { Jones, Mothersbaugh e Beatty (2000) e Edward e } \\
\text { Sahadev (2011) }\end{array}$ \\
\cline { 2 - 3 } Retenção de Clientes & RUSTR_4 & Bansal, Irving e Taylor (2004) e Dagger e David (2012) \\
\hline
\end{tabular}

Fonte: Elaborada pelos autores

Obs.: Item de escala com escore reverso: RETEN_5.

O passo seguinte foi a realização da validação de conteúdo (KINNEAR; TAYLOR, 1996). Para tanto, o instrumento de coleta de dados foi submetido a três experts da área e a um pré-teste aplicado a oito clientes dos planos de saúde coletivos (empresariais). Salienta-se que, optou-se pela abordagem de forma direta na coleta de dados, sendo utilizando o método de auto-preenchimento (FINK, 2013). Cabe salientar que a coleta de dados foi realizada entre os meses de junho e agosto de 2013.

\subsection{Procedimentos da análise dos dados}

A etapa de preparação dos dados foi realizada com a intenção de verificar as características e o ajuste dos dados coletados, com a intenção de identificar os dados omissos (missings), bem como as observações atípicas (outliers). Para a verificação dos missings foi utilizada a técnica de listwise deletion (DAVEY; SAVLA, 2010), pela qual se considera somente os questionários com dados completos. Assim, três questionários (casos) foram eliminados, totalizando 283 questionários válidos. A seguir, foram verificados os outliers, utilizando uma combinação de análises uni e multivariadas (ENDERS, 2010). Primeiramente, foi aplicada a verificação dos escores padrão ( $Z$ scores) univariados, sendo que 13 casos foram eliminados por apresentaram valores superiores a |3| (HAIR Jr. et al., 2009). Também foram investigados os outliers multivariados a partir do cálculo da distância de Mahalanobis $\left(D^{2}, p<0,005\right.$ e $\left.g l=30\right)$, considerando os valores com índices de até $3\left(\mathrm{D}^{2} / \mathrm{gl}\right)$, sendo que apenas um caso apresentou um índice de 3,22 e, por isso, eliminado da base de dados, totalizando 269 casos válidos.

Na sequência, foram realizados os testes das suposições da análise mutivariadas, através do teste da normalidade pro meio do teste de (skewness), que apresentou os valores entre -0,263 e 1,379; e da kurtosis, sendo que os valores variaram de $-0,515$ a 3,555. Já a homoscedasticidade foi testada através do Teste de Levene, pelo qual se observou que as variáveis métricas apresentaram um padrão de heteroscedasticidade somente em uma das variáveis. Para a linearidade foram testados os Coeficientes de Correlação de Pearson, sendo que os coeficientes apresentaram valores positivos, menores que 0,90 e com significância $p<0,001$, demonstrando a linearidade dos dados. Também foi verificada a multicolinearidade através do teste de Valor de Tolerância deve onde os valores variaram entre 0,20 e 0,80, e para o Fator de Inflação da Variância (VIF - Variance Inflation Factor), as variáveis apresentaram valores entre 1,25 e 5,05, sendo que seguiram os valores recomendados na literatura (HAIR Jr. et al., 2009; MEYERS; GAMST; GUARINO, 2013).

\section{Apresentação dos resultados}

\subsection{Caracterização da amostra}

Considerando os critérios de seleção da amostra, os cargos mais evidenciados dos participantes foram: Analista de Recursos Humanos (21,19\%), Coordenador de Recursos Humanos (14,13\%), Analista Administrativo (10,78\%), Diretor Administrativo (6,69\%), Diretor de Recursos Humanos (5,58\%), Gerente Administrativo (4,83 \%) e Auxiliar de Departamento Pessoal (4,83\%), totalizando 68,03\% da amostra. 


\subsection{Validação individual dos construtos}

A validação individual de um construto mede o quanto um conjunto de variáveis medidas representa o construto latente (HAIR Jr. et al., 2009). Para a validação individual dos construtos foram avaliadas a unidimensionalidade, a confiabilidade, a validade convergente e a validade discriminante. Aunidimensionalidade foi realizada a Análise Fatorial Exploratória (AFE), por meio de componentes principais e através da rotação ortogonal Varimax (AFIFI; MAY; CLARK, 2012), sendo que as cargas fatoriais da AFE variaram entre 0,578 e a 0,850, sendo consideradas satisfatórias (HAIR Jr. et al., 2009), obtendo uma variância explicada de no mínimo de 79,18\%. Além disso, foi verificado o Alpha de Cronbach, que apresentou valor mínimo de 0,727, sendo superior ao valor recomendado na literatura (valores iguais ou superiores a 0,70) (HAIR Jr. et al., 2009). Dando sequência na validação dos construtos, foi calculada a validade convergente por meio da Análise Fatorial Confirmatória (AFC), considerando como parâmetros valores acima de 0,5 (KLINE, 2011), conforme Tabela 2.

Tabela 2 - Alpha de Cronbach, confiabilidade composta e variância extraída

\begin{tabular}{lccc}
\hline Construtos & Alpha de Cronbach & Confiabilidade Composta & Variância Extraída \\
\hline Reputação do Provedor de & 0,727 & 0,826 & 0,570 \\
Serviços & 0,774 & 0,834 & 0,560 \\
Custos de Troca & 0,818 & 0,756 & 0,510 \\
Retenção de Clientes & & & \\
\hline
\end{tabular}

Fonte: Elaborada pelos autores.

A validade discriminante, por sua vez, foi testada através do cálculo das variâncias compartilhadas, considerando o método proposto por Fornell e Larcker (1981), no qual as variâncias extraídas dos construtos são comparadas com as variâncias compartilhadas, de acordo com a Tabela 3. Os resultados apresentaram uma validade discriminante adequada dos três construtos em análise.

Tabela 3 - Validade discriminante

\begin{tabular}{lccc}
\hline Construtos & $\begin{array}{c}\text { Reputação do Provedor } \\
\text { de Serviços }\end{array}$ & Custos de Troca & $\begin{array}{c}\text { Retenção de } \\
\text { Clientes }\end{array}$ \\
\hline Reputação do Provedor de Serviços & 0,742 & & \\
Custos de Troca & 0,260 & 0,560 & \\
Retenção de Clientes & 0,732 & 0,309 & 0,510 \\
\hline
\end{tabular}

Fonte: Elaborada pelos autores.

Ao se analisar os resultados, é possível observar que há validade discriminante entre os construtos, pois a variância extraída é maior que a variância compartilhada em todas as relações.

\subsection{Validação do modelo teórico}

Ao se validar o ajuste geral do Modelo Teórico proposto é verificada a similaridade entre as matrizes de covariância estimada e observada, através das medidas de qualidade de ajuste GOFs (Goodnes-of-fit), as quais são classificadas em medida de ajuste absoluto (GFI e RMSEA); medidas de ajuste incremental (AGFI, TLI e NFI); e medida de ajuste parcimonioso (CFI) (BYRNE, 2010; WEST; TAYLOR; WU, 2012; BLUNCH, 2013).

No presente estudo, ao se analisar as medidas de ajuste, observa-se que o GFI $(0,902)$ e CFI $(0,903)$ foram satisfatórios. O RMSEA $(0,075)$ também apresentou valores conforme recomendado na literatura estando entre 0,05 e 0,08 sendo adequado aos parâmetros recomendados (HAIR Jr. et al., 2009; KLINE, 2011). Porém, as demais medidas AGFI $(0,859)$, NFI $(0,851)$ e TLI $(0,878)$ apresentarem valores na zona de fronteira por estarem muito próximos a 0,90 (HAIR Jr. et al., 2009; KLINE, 2011), conforme apresentado na Tabela 4. 
Tabela 4 - Medidas de ajustes do modelo

\begin{tabular}{c|c}
\hline Medidas de Ajustes do Modelo & Índices ou Valores Obtidos para as Medidas de Ajuste do Modelo \\
\hline RMSEA & 0,075 \\
CFI & 0,903 \\
TLI & 0,878 \\
NFI & 0,851 \\
GFI & 0,902 \\
AGFI & 0,859 \\
\hline
\end{tabular}

Fonte: Elaborada pelos autores.

Avançando na validação do modelo, foi realizado o teste de hipóteses, o qual examina a significância e a magnitude dos coeficientes de regressão estimados (HAIR Jr. et al., 2009; AFIFI; MAY CLARK, 2012). Por conseguinte, a Tabela 5 apresenta os caminhos estruturais, os coeficientes não padronizados, os errospadrão, os coeficientes padronizados, os $t$-values e as probabilidades.

Tabela 5 - Teste de hipóteses

\begin{tabular}{l|l|l|l|l|l|l|l}
\hline $\mathrm{H}_{\mathrm{i}}$ & $\begin{array}{l}\text { Caminhos } \\
\text { Estruturais }\end{array}$ & $\begin{array}{l}\text { Coeficientes Não } \\
\text { Padronizados (b) }\end{array}$ & Erros & $\begin{array}{l}\text { Coeficientes } \\
\text { Padronizados }(\beta)\end{array}$ & t-values & $\mathrm{p}$ & Resultados \\
\hline $\mathrm{H}_{1}$ & REPUT $\rightarrow$ CUSTR & 0,108 & 0,098 & 0,095 & 1,096 & 0,273 & $\begin{array}{l}\text { Não } \\
\text { Suportada }\end{array}$ \\
\hline $\mathrm{H}_{2}$ & REPUT $\rightarrow$ RETEN & 1,341 & 0,136 & 0,813 & 9,873 & 0,000 & Suportada \\
\hline $\mathrm{H}_{3}$ & CUSTR $\rightarrow$ RETEN & 0,241 & 0,092 & 0,165 & 2,625 & 0,009 & Suportada \\
\hline
\end{tabular}

Fonte: Elaborada pelos autores

Obs.: Nível de significância de 0,05.

De acordo com os resultados apresentados, observa-se que as hipóteses $H_{2}$ (a reputação do provedor de serviços influencia positivamente a retenção de clientes, $\beta=0,813, p<0,001$ ) e a $H_{3}$ (os custos de troca afeta positivamente a retenção dos clientes, $\beta=0,165, p<0,001$ ), foram suportadas estatisticamente. Por outro lado, a hipótese $H_{1}$ (a reputação do provedor de serviços influencia positivamente os custos de troca, $\beta=0,095, p=0,273$ ) não foi suportada estatisticamente.

Com a intenção de ampliar a verificação da efetividade do teste de hipóteses, foram analisados os coeficientes de determinação $\left(R^{2}\right)$, os quais têm por premissa de que quanto maior o poder de explicação da equação da regressão, melhor será a predição da variável dependente (HAIR Jr. et al., 2009). Os coeficientes de determinação $\left(R^{2}\right)$ do Modelo Teórico são apresentados na Tabela 6.

Tabela 6 - Coeficientes de Determinação

\begin{tabular}{c|c}
\hline Construtos & Coeficientes de Determinação $\left(\mathrm{R}^{2}\right)$ \\
\hline Custos de Troca & 0,009 \\
\hline Retenção de Clientes & 0,713 \\
\hline
\end{tabular}

Fonte: Elaborada pelos autores.

Para tanto, o modelo estrutural apresentou o seguinte resultado: $71,3 \%$ da variância da retenção de clientes pode ser explicada por suas variáveis independentes, sendo elas, os custos de troca e a reputação do provedor de serviços, o que denota um alto poder explicativo (HAIR Jr. et al., 2009; MEYERS; GAMST; GUARINO, 2013).

\subsection{Análise da mediação dos custos de troca}

De acordo com Vieira (2009), a variável mediadora influencia a relação entre a variável independente e a variável dependente, de modo que a sua inserção na equação estrutural neutraliza ou bloqueia a força do impacto da variável independente sobre a dependente. 
No presente estudo, ao se testar a mediação proposta na $\mathrm{H}_{4}$, foi utilizada a técnica de Análise de Regressão Linear, sendo estes os procedimentos recomendados por Zhao, Lynch Jr. e Chen (2010), Preacher e Hayes (2004) e Hayes (2013), sendo que os autores sugerem que, ao se analisar a mediação, o efeito indireto da variável independente na variável dependente deve ser significativo. Diante disso, a mediação ocorre mesmo que o efeito total (c') seja insignificante (ZHAO; LYNCH Jr.; CHEN, 2010). O procedimento proposto por Zhao, Lynch Jr. e Chen (2010) é embasado nas proposições de Preacher e Hayes (2004) e Hayes (2013), os quais sugerem o uso do bootstraping para estimação da variação do efeito indireto, o qual é considerado significante quando o intervalo de confiança (95\%) não contiver zero, ou seja, o intervalo não contém um efeito nulo. Os autores consideram, ainda, que a mediação é completa quando o efeito direto da variável independente na variável dependente é insignificante com a presença do mediador, sendo esta parcial quando o efeito for significativo. Considerando as implicações relatadas, a análise de mediação, no presente estudo, seguiu os procedimentos comentados anteriormente, conforme Figura 2.

Figura 2 - Mediação dos custos de troca

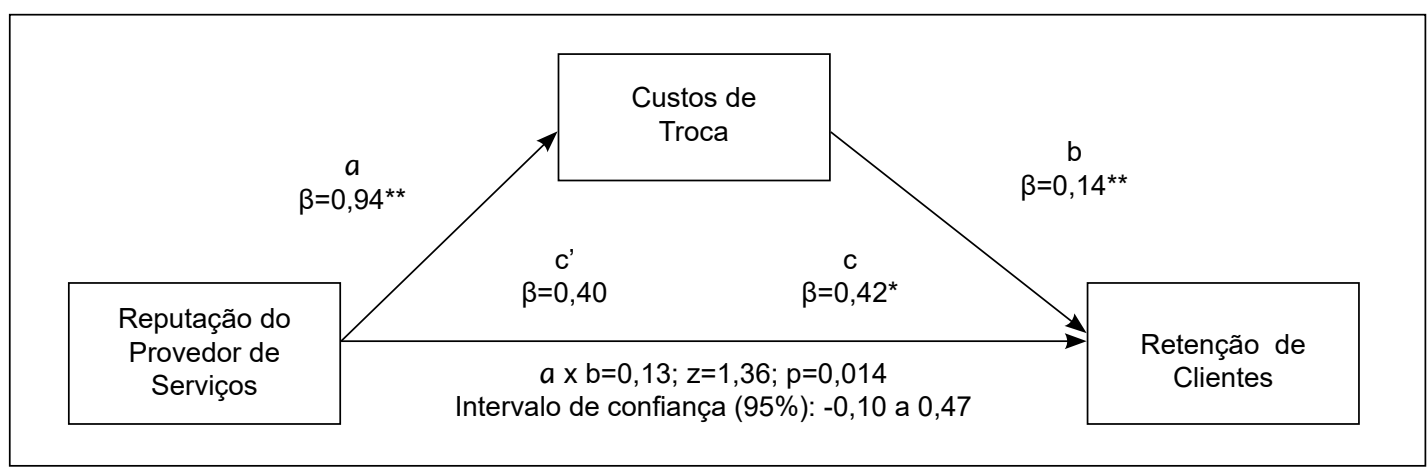

Fonte: Elaborada pelos autores

Obs.: Os valores apresentados não são padronizados. ${ }^{* *} p<0,01$.

Neste estudo, a reputação do provedor de serviços foi testada como variável independente, os custos de troca como variável mediadora e a retenção de clientes como variável dependente. Percebe-se, que o caminho entre a variável independente e a variável mediadora não foi significativo ( $\alpha=0,94 ; t=1,37 ; p=0,300)$. Já a relação entre a variável mediadora e a variável dependente foi significativa $(b=0,145 ; t=4,238 ; p=0,000)$. O efeito indireto da reputação do provedor de serviços sobre a retenção de clientes mediada pelos custos de troca também foi significativo ( $a \times b=0,013 ; z=1,36 ; p=0,014$ ). Além disso, o intervalo de confiança (95\%) para o efeito indireto, calculado por meio de 5.000 reamostragens no procedimento de bootstraping, não inclui zero ou feito nulo $(-0,10$ a 0,47$)$. $O$ efeito total da reputação do provedor de serviços sobre a retenção de clientes foi significativo $(c=0,42 ; t=8,234 ; p=0,000)$, da mesma forma que o efeito direto da reputação do provedor de serviços sobre a retenção de clientes $\left(c^{\prime}=0,40, t=8,21 ; p=0,000\right)$. Estes resultados evidenciam que os custos de troca não desempenham um papel mediador na relação entre a reputação do provedor de serviços e a retenção de clientes, não suportando a $\mathrm{H}_{4}$.

\section{Considerações finais}

Ao se fazer uma verificação na literatura, constatou-se que existem diversos estudos que abordam a retenção de clientes, porém não há um consenso em termos da definição dos seus determinantes, salientado a necessidade do desenvolvimento de novas pesquisas com a intenção de verificar, com mais precisão, quais são os determinantes que impactam na continuidade dos relacionamentos com os clientes existentes. Diante disso, esta pesquisa abordou as proposições e indicações de pesquisas futuras dos principais estudos relativos à retenção de clientes, considerando como seus determinantes a reputação do provedor de serviços e os custos de troca, além de verificar a síntese dos trabalhos mais referenciados a respeito dos construtos abordados no contexto da manutenção e da ampliação dos relacionamentos, visando trazer acréscimos em se tratando da prestação de serviços e seu impacto teórico e gerencial sobre a retenção de clientes. 
Considerando a comprovação das hipóteses $\mathrm{H}_{2}$ (a reputação do provedor de serviços influencia positivamente a retenção de clientes) e a $\mathrm{H}_{3}$ (os custos de troca influenciam positivamente a retenção de clientes) do Modelo Teórico proposto, destaca-se a importância de testar as relações entre a reputação do provedor de serviços e os custos de troca no âmbito das relações B2B, principalmente em contextos inerentes às empresas prestadoras de serviços mais técnicos ou complexos (MOLINARI; ABRATT; DION, 2008; TOUFAILY; RICARD; PERRIEN, 2013), como é o caso dos serviços de saúde, que serviram de pano de fundo desta pesquisa.

Isto de justifica uma vez que os clientes de planos de saúde costumam permanecer em um relacionamento com o provedor de serviços quando percebem poucas alternativas ou quando a concorrência oferecer serviços limitados e tiver poucos profissionais em algumas especializações. Isto posto, verifica-se que os sacrifícios financeiros, de tempo, físicos e emocionais percebidos são fatores que dificultam a procura de um novo provedor de serviços, servindo como uma barreira à troca (FOWDAR, 2013; BERRY, BENDAPUDI, 2007), fazendo com que os clientes permaneçam com o provedor de serviços atual. Tal situação se sustenta, ainda, pela evidência de que os custos de troca resultam em um maior risco para o cliente porque podem representar sacrifícios e/ou penalidades que os clientes percebem, podendo incorrer em despesas monetárias e não monetárias, tais como tempo e outros tipos de esforços em trocar para um novo provedor de serviços (DAGGER; DAVID, 2012).

Em contrapartida, a não confirmação da $\mathrm{H}_{1}$ (a reputação do provedor de serviços influencia positivamente os custos de troca), evidencia que, em termos teóricos, existem evidências de que a reputação do provedor de serviços esteja relacionada aos custos de troca (HELGESEN; NESSET, 2007; HAN; HYUN, 2013), porém, os resultados ainda precisam ser testados com maior amplitude para que se construam conclusões mais definitivas, pois alguns trabalhos como o de Han e Hyun (2013) apresentam a reputação com uma influência moderada sobre os custos de troca, embora os autores ressaltem que esta relação é um preditor forte das respostas atitudinais e comportamentais dos clientes em relação ao provedor de serviços.

Neste caso, Fowdar (2013) destaca que apesar de o construto custos de troca ser testado desde o início da década de 90, é pequeno o volume de estudos que investigam os fatores sobre o porquê os clientes não trocam de provedores de serviços, mesmo que, em determinada situação, não estejam sendo supridas suas necessidades, desejos ou expectativas. Inclusive, Lacey (2012) aponta que a relação positiva entre os custos de troca e a retenção de clientes, em alguns serviços, como, por exemplo, na área de serviços da saúde, não ocorre com a mesma intensidade em outros serviços. Esta influência parece estar ligada ao grau de envolvimento dos clientes com os serviços prestados, que é alto no caso de serviços de saúde.

Em se tratando, da não confirmação da $\mathrm{H}_{4}$, que testou a mediação dos custos de troca na relação entre a reputação do provedor de serviços e a retenção de clientes, não confirmou o pressuposto apresentado na meta-análise realizada por Palmatier et al. (2006), que sinaliza os custos de troca como um mediador-chave nas trocas relacionais. Uma explicação para este resultado pode ser a diversidade existente nos diferentes ambientes de serviços e suas especificidades, o que também demanda novas investigações.

Em resumo, o presente estudo contribui, efetivamente, para que se encontrem relações significativas entre a reputação do provedor de serviços e os custos de troca como construtos determinantes da retenção de clientes, que podem vir a resultar em maior lucratividade e rentabilidade e, consequentemente, maior competitividade para os provedores de serviços, mais especificamente, no contexto corporativo.

Os resultados da presente pesquisa desperta a necessidade de se propor novos estudos em torno da retenção de clientes e seus determinante, com a intenção de melhor compreender as relações desenvolvidas no modelo teórico que afetam a continuidade dos relacionamentos entre os clientes e as empresas prestadoras de serviços. Para tanto, sugere-se o desenvolvimento de novas pesquisas, através da replicação do modelo teórico proposto no contexto B2C - Business-to-Consumer e, em outros ambientes de serviços, como serviços financeiros e de telefonia. Destaca-se, ainda, a possibilidade de se fazer o refinamento da escala da reputação em provedores de serviços e dos custos de troca por meio da inclusão de novos itens ou da adaptação das escalas atuais em diferentes contextos organizacionais. Por fim, quanto à especificação do modelo, também seria possível testar os custos de troca como mediador entre outros construtos, como, por exemplo, a confiança e o valor percebido e seu impacto sobre a retenção de clientes. 


\section{Referências}

AFIFI, A.; MAY, S.; CLARK, V. A. Practical multivariate analysis. $5^{\text {th }}$ edition. Taylor \& Francis Group, 2012.

ANG, S. H.; WIGHT, A. Building intangible resources: the stickiness of reputation. Corporate Reputation Review, v. 2, p. 21-32, 2009.

AURIER, P.; N'GOALA, G. The differing and mediating roles of trust and relationship commitment in service relationship maintenance and development. Journal of the Academic Marketing Science, v. 38, n. 3, p. 303-325, 2010.

AULA, P.; MANTERE, S. Making and breaking sense: an inquiry into the reputation change. Journal of Organizational Change Management, v. 26, n. 2, p. 340-352, 2013.

BANSAL, H. S.; IRVING, P. G.; TAYLOR, S. F. A three-component model of customer commitment to service providers. Journal of the Academy of Marketing Science, v. 32, n. 3, p. 234-250, 2004.

BARNETT, M. L.; JERMIER, J. M.; LAFFERTY, B. A. Corporate reputation: the definitional landscape. Corporate Reputation Review, v. 9, n. 1, p. 26-38, 2006.

BERRY, L.; BENDAPUDI, N. Health care: a fertile field for service research. Journal of Service Research, v. 10, n. 2, p. 111-122, 2007.

BLUNCH, N. J. Introduction to structural equation modeling using IBM SPSS statistics and AMOS. $2^{\text {nd }}$ edition. Thousand Oaks: Sage Publications, 2013.

BRUSH, T. H.; DANGOL, R.; O'BRIEN, J. P. Customer capabilities, switching costs, and bank performance. Strategic Management Journal, v. 13, n. 13, p. 1.499-1.515, 2012.

BURNHAM, T. A.; FRELS, J. K; MAHAJAN, V. Consumer switching costs: a typology antecedents, and consequences. Journal of the Academy of Marketing Science, v. 31, n. 2, p. 109-126, 2003.

BYRNE, B. M. Structural equation modeling with AMOS: basic concepts applications, and programming. $2^{\text {nd }}$ edition. New York: Routledge, 2010.

CHACHAL, H.; BALA, M. Significant components of service brand equity in healthcare sector. International Journal of Health Care Quality Assurance, v. 25, n. 4, p. 343-362, 2012.

CLEMES, M. D.; GAN, C.; ZHANG, D. Customer switching behavior in the Chinese retail banking industry. International Journal of Bank, v. 28, n. 7, p. 519-546, 2010.

DAGGER, T. S.; DAVID, M. E. Uncovering the real effect of switching costs on the satisfaction-loyalty association: the critical role of involvement and relationship benefits. European Journal of Marketing, $v$. 36, n. 3/4, p. 447-468, 2012.

DAVEY, A.; SAVLA, J. Statistical power analysis with missing data: a structural equation modeling approach. New York: Routledge, 2010.

DONEY, P.; CANNON, J. P. An examination of the nature of trust in buyer-seller relationships. Journal of Marketing, v. 61, n. 2, p. 35-51, 1997.

EDWARD, M.; SAHADEV, S. Role of switching costs in the service quality, perceived value, customer satisfaction and customer retention linkage. Asia Pacific Journal of Marketing and Logistics, v. 22, n. 3, p. 327-345, 2011.

ENDERS, C. K. Applied missing data analysis. New York: The Guilford Press, 2010.

FINK, A. How to conduct surveys: a step-by-step guide. $5^{\text {th }}$ edition. Thousand Oaks: Sage Publications, 2013.

FOMBRUN, C. J. Reputation: realizing value from the corporate image. Boston: Harvard Business School Press, 1996. 
FORNELL, C.; LARCKER, D. Evaluating structural equation models with unobserved variables and measurement error. Journal of Marketing Research, v. 18, n. 1, p. 39-50, 1981.

FOWDAR, R. R. R. Assessing the influence of switching barriers on patients' expectations and tolerance zone. International Journal of Health Care Quality Assurance, v. 26, n. 2, p. 226-249, 2013.

GANESAN, S. Determinants of long-term orientation in buyer-seller relationships. Journal of Marketing, n. 2, v. 58, p. 1-19, 1994.

GANESH, J.; ARNOLD, M. J.; REYNOLDS, K. Understanding the customer base of service providers: an examination of the differences between switchers and stayers. Journal of Marketing, v. 64, n. 3, p. 65$87,2000$.

GÓMEZ, J.; MAÍCAS, J. P. Do switching costs mediate the relationship between entry timing and performance? Strategic Management Journal, v. 32, n. 12, p. 1251-1269, 2011.

GÖZÜKARA, I.; YILDIRIM, O. Affective commitment and corporate reputation. The effects of attitude toward advertising and trust. Academic Journal of Economic Studies, v. 1, n. 3, p. 126-147, 2015.

GRAHAM, M. E.; BANSAL, P. Consumers' willingness to pay for corporate reputation: the context of airline companies. Corporate Reputation Review, v. 10, n. 3, p. 189-200, 2007.

GRZYBOWSKI, L. Estimating switching costs in mobile telephony in the U.K. Journal of Industry, Competition and Trade, v. 8, n. 2, p. 113-132, 2008.

HAIR Jr., J. F.; BLACK,W. C.; BABIN, B. J.; ANDERSON, R. E.; TATHAM, R. L. Análise multivariada de dados. 6. ed. Porto Alegre: Bookman, 2009.

HAN, H.; HYUN, S. S. Image congruence and relationship quality in predicting switching intention: consyicuousness of product use as a moderator variable. International Journal of Hospitality Management, v. 37, n. 3, p. 303-329, 2013.

HAYES, A. F. Introduction to mediation, moderation, and conditional process analysis: a regression-based approach. New York: The Guilford Press, 2013.

HELGESEN, O.; NESSET, E. Images, satisfaction and antecedents: drivers of student loyalty? A case study of a Norwegian University College. Corporate Reputation Review, v. 10, n. 1, p. 38-59, 2007.

HELM, S. The role of corporate reputation in determining inventor satisfaction and loyalty. Corporate Reputation Review, v. 10, n. 1, p. 22-37, 2007.

HODOVIC-BABIC, V.; MEHIC, E.; ARSLANAGIC, M. Influence of banks corporate reputation on organizational buyers perceived value. Procedia - Social and Behavioral Sciences, v. 24, p. 351-360, 2011.

HOYLE, R. H. Assumptions in structural equation modeling. In: HOYLE, R. H. (Ed.). Handbook of structural equation modeling. New York: The Guilford Press, 2012. chapter 8, p. 126-144.

JAWORSKI, B. J.; KOHLI, A. K. Market orientation: antecedents and consequences. Journal of Marketing, v. 57, n. 3, p. 53-71, 1993.

JOHNSON, D.; GRAYSON, K. Cognitive and affective trust in service relationship. Journal of Business Research, v. 58, n. 4, p. 500-507, 2005.

JONES, M. A.; MOTHERSBAUGH, D. L.; BEATTY, S. E. Switching barriers and repurchase intentions in services. Journal of Retailing and Consumer Services, v. 76, n. 2, p. 259-274, 2000.

JONES, M. A.; REYNOLDS, K. E.; MOTHERSBAUGH, D. L.; BEATTY, S. E. The positive and negative effects of switching costs on relational outcomes. Journal of Service Research, v. 9, n. 4, p. 335-355, 2007.

JOSE, P. E. Doing statistical mediation \& moderation. New York: The Guilford Press, 2013.

KAMAKURA, W. A.; WEDEL, M.; ROSA, F.; MAZZON, J. A. Cross-selling through database marketing: 
a mixed factor analyzer for data augmentation and prediction. International Journal of Research in Marketing, v. 20, n. 1, p. 45-65, 2003.

KIM, M. S.; AHN, J. H. Comparison of trust sources of an online market-maker in the e-marketplace: buyer's and seller's perspectives. The Journal of Computer Information Systems, v. 47, n. 1, p. 84-94, 2006.

KLINE, R. B. Principles and practice of structural equation modeling. New York: The Guilford Press, 2011.

KONUK, F. A.; KONUK, F. The relationship between service quality, economic and switching costs in retail banking. Business Systems Review, v. 2, n. 1, p. 1-16, 2013.

LACEY, R. How customer voice contributes to stronger service provider relationships. Journal of Services Marketing, v. 26, n. 2, p. 137-144, 2012.

LOUREIRO, S. M. C.; KASTENHOLZ, E. Corporate reputation, satisfaction, delight, and loyalty towards rural lodging unit in Portugal. International Journal of Hospitality Management, v. 30, p. 575-583, 2011.

MALHOTRA, N. K.; BIRKS, D.; WILLS, P. Marketing research: applied approach. $4^{\text {th }}$ edition. New York: Pearson, 2012.

MEYERS, L. S.; GAMST, G. C.; GUARINO, A. J. Applied multivariate research: design and interpretation. $2^{\text {nd }}$ edition. Thousand Oaks: Sage Publications, 2013.

MILAN, G. S.; CAMARGO, M. E.; DE TONI, D.; PINTO, M. M. B.; COSTA, C. A. The relational practice existing between a service provider and its customers and the customer retention: a comparison between a theoretical model an rival. Australian Journal of Basic and Applied Sciences, v. 7, n. 7, p. 904-915, 2013.

MILAN, G. S.; DE TONI, D. A construção de um modelo sobre a retenção de clientes e seus antecedentes em um ambiente de serviço, REAd - Revista Eletrônica de Administração, v. 18, n. 2, p. 433-467, 2012.

MILAN, G. S.; PRESTES, A. R.; MUNHOZ, L. P. S. Análise do valor do cliente (lifetime value): um estudo ambientado em uma operadora de planos de saúde. BASE - Revista de Administração e Contabilidade da Unisinos, v. 7, n. 2, p. 123-134, 2010.

MOLINARI, L. K.; ABRATT, R.; DION, P. Satisfaction, quality and value and effects on repurchase and positive word-of-mouth behavioral intentions in a B2B services context. Journal of Services Marketing, v. 22, n. 5, p. 363-373, 2008.

N'GOALA, G. Customer switching resistance (CSR): the effects of perceived equity, trust and relationship commitment. International Journal of Service Industry Management, v. 18, n. 5, p. 510-533, 2007.

OLIVER, R. L. Satisfaction: a behavioral perspective on the consumer. $2^{\text {nd }}$ edition. New York: M. E. Sharpe, 2010.

OU, W.; SHIH, C.; CHEN, C. Antecedents and consequences of relationship commitment: an empirical study in Taiwan. Journal of Commerce and Management, v. 24, n. 3, p. 228-242, 2014.

PALMATIER, R. W.; DANT, R. P.; GREWAL, D.; EVAN, K. R. Factors influencing the effectiveness of relationship marketing: a meta-analysis. Journal of Marketing, v. 70, n. 4, p. 136-153, 2006.

PATTERSON, P. G. A contingency model of behavioural intentions in a services context. European Journal of Marketing, v. 38, n. 9/10, p. 1304-1315, 2004.

PHILIPPE, D.; DURAND, R. The impact of norm-conforming behaviors on firm reputation. Strategic Management Journal, v. 32, n. 9, p. 939-969, 2011.

PIVA, L. C.; FUMAGALLI, L. A. W.; BAPTISTA, P. P.; SILVA, W. V. Relação entre satisfação, retenção e rentabilidade de cliente no setor de planos de saúde. RCA - Revista de Ciências da Administração, v. 9, n. 19, p. 54-80, 2007. 
PREACHER, K. J.; HAYES, A. F. SPSS and SAS procedures for estimating indirect effects simple mediation models. Behavior Research Methods, Instruments, and Computers, v. 36, p. 717-731, 2004.

REICHHELD, F. F. The loyalty effect: the hidden force behind growth, profits, and lasting value. Boston: Harvard Business Scholl Press, 1996.

RIVERS, P. A.; GLOVER, S. H. Healthcare competition, strategic mission, and patient satisfaction: research model and proposition. Journal of Health Organization and Management, v. 22, n. 6, p. 627641, 2008.

ROOS, I.; GUSTAFSSON, A. Understanding frequent switching patterns: a crucial element in managing customer relationship. Journal of Service Research, v. 10, n. 1, p. 93-108, 2007.

SANTOS, C. P.; FERNANDES, D. V. H. A recuperação do serviço como ferramenta de relacionamento e seu impacto na confiança e lealdade. RAE - Revista de Administração de Empresas, v. 48, n. 1, p. 1024, 2008.

SEO, D.; RANGANATHAN, D. B.; BABAD, Y. Two-level mode of customer retention in the US mobile telecommunications service market. Telecommunications Policy, v. 32, n. 3/4, p. 182-196, 2008.

SIERRA, J. J.; McQUITTY, S. Service providers and customers: social exchange theory and service loyalty. The Journal of Services Marketing, v. 19, n. 6/7, p. 392-400, 2005.

TOUFAILY, E.; RICARD, L.; PERRIEN, J. Customer loyalty to a commercial website: descriptive metaanalysis of the empirical literature proposal of an integrative mode. Journal of Business Research, $v$. 66, n. 9, p. 1.436-1.447, 2013.

TYRAN, K. L.; GIBSON, C. B. Is what you see, what you get? The relationship among surface-and deep-level heterogeneity characteristics, group efficacy, and team reputation. Group Organization Management, v. 33, n. 1, p. 46-76, 2008.

VASUDEVAN, H.; GAUR, S. S.; SHINDE, R. K. Relational switching costs, satisfaction and commitment: a study in the Indian manufacturing context. Asia Pacific Journal of Marketing and Logistics, v. $18 \mathrm{n}$. 4, p. 342-353, 2006.

VIEIRA, V. A. Moderação, mediação, moderadora-mediadora e efeitos indiretos em modelagem de equações estruturais: uma aplicação no modelo de desconfirmação de expectativas. RAUSP - Revista de Administração, v. 44, n. 1, p. 17-33, 2009.

WALKER, K. A systematic review of the corporate reputation literature: definition, measurement, and theory. Corporate Reputation Review, v. 12, n. 4, p. 357-387, 2010.

WALSH, G.; DINNIE, K.; WIEDMANN, K. How do corporate reputation and customer satisfaction impact customer defection? A study of private energy customers in Germany. The Journal of Services Marketing, v. 20, n. 6, p. 412-420, 2006.

WEST, S. G.; TAYLOR, A. B.; WU, W. Model fit and model selection in structural equation modeling. In: HOYLE, R. H. (Ed.). Handbook of structural equation modeling. New York: The Guilford Press, 2012. chapter 13, p. 209-231.

WONG, C. B. The Influence of customer satisfaction and switching costs on customer retention: retail internet banking services. Global Economy and Finance Journal, v. 4, n. 1, p. 1-18, 2011.

ZHAO, X.; LYNCH Jr., J. G.; CHEN, Q. Reconsidering Baron and Kenny: myths and truths about mediation analysis. Journal of Consumer Research, v. 37, n. 2, p. 197-206, 2010.

Submetido em: 01/11/2018

Aprovado em: 02/12/2019 